\title{
Mechanism of electron cloud clearing in the accumulator ring of the Spallation Neutron Source
}

\author{
L. F. Wang, D. Raparia, J. Wei, and S. Y. Zhang \\ Brookhaven National Laboratory, Upton, New York 11973-5000, USA
}

(Received 3 December 2003; published 2 March 2004)

\begin{abstract}
We describe a mechanism using clearing electrodes and solenoids to remove the electron cloud in the Spallation Neutron Source's accumulator ring. Simulation indicates that strong multipacting could happen at median clearing fields. A similar phenomenon was reported in an experimental study at Los Alamos Laboratory's Proton Storage Ring. A weak solenoid is proven to be a good remedy for suppressing multipacting by confining electrons near the chamber wall's surfaces. We investigated the clearing mechanism using both electrodes and solenoids.
\end{abstract}

DOI: 10.1103/PhysRevSTAB.7.034401

PACS numbers: 29.27.Bd, 29.20.Dh, 29.85.+c

\section{INTRODUCTION}

Electron cloud instability was first reported at The Budker Institute of Nuclear Physics Proton Storage Ring (PSR) in 1965 [1]. In 1971, an electron cloud caused beam instability was observed at CERN Intersecting Storage Rings during a coasting-beam operation and was cured with clearing electrodes [2,3]. In 1988, it also was observed for a bunched beam in the PSR at Los Alamos National Laboratory (LANL) [4]. Such instability, which seems to reflect the interaction of electrons and protons, also occurs at the Alternating Gradient Synchrotron booster [5], and, similarly, was reported in several other machines: KEK's Photo Factory $[6,7]$ and $B$ Factory (KEKB) [8], Stanford Linear Accelerator Center's PEP-II [9], Beijing's Electron Positron Collider [10], and the European Organization for Nuclear Research's Proton Synchrotron (PS) and Super Proton Synchrotron (SPS) [11,12]. Apparently, bunched beaminduced multipacting causes electrons to accumulate inside the vacuum chamber $[13,14]$ that then interact with the proton or positron beam, causing its instability. Experimental observations of electron cloud instabilities are distinctively different for "short bunches," where multibunch multipacting is expected to be important (PS, SPS, and $B$ factories) and for "long bunches," where single-bunch, trailing-edge multipacting is expected to dominate [15]. For the coasting beam, the electrons are accumulated at the chamber center due to the trapping of beam potential instead of beam-induced multipacting. Spallation Neutron Source's (SNS) beam is a bunched beam where the electron cloud is mainly produced by multipacting.

For short bunches, the buildup of the electron cloud depends on the multibunch multipacting. Therefore, the clearing effects depend on the spacing of the bunches. The requirement for the clearing electrode or solenoid is to clear the electrons within one bunch spacing. Because there is no beam space-charge force in the bunch gap, an electron can easily drift from one side of the chamber's surface to the other side under the force of a clearing field or a solenoid field. A weak solenoid field drives electrons into making a half-circle orbit so that they hit the wall's surfaces after gyrating for about half a period. Bruning and Zimmermann studied a multiwires clearing electrode system [16,17] and Wang et al. suggested it might be used in the KEKB [18]. The wires are set parallel with the beam's pipe so that they excite a transverse clearing field. The clearing field near the wall's surface turns back the electrons by retarding their velocity. This system has notable merit because it works in both the drift region and the magnetic region, whereas a solenoid can be applied only in a magnetic-field-free region. Further, it requires a low clearing voltage and contributes only to small impedance due to its small diameter compared with the ionclearing electrode system. Caspers suggested one type of "invisible clearing electrode" with negligible impedance [19], viz., an alumina body coating with a high-resistive thin layer. For the wake field, this electrode is transparent.

For long bunches, such as those at the SNS, electron cloud multipacting is a single-bunch phenomenon in which strong multipacting occurs at the tail of the bunch. Because of the beam's space-charge effect, clearing the electrons is more complicated than for short bunches. For example, the orbit of an electron in the presence of a solenoid is not a simple half circle due to the combined effects of the beam's space-charge field and the solenoid's field. Thus, although a solenoid has been proven both experimentally and by simulation to be a good cure for electron cloud buildup in the drift region for a short bunch, such as $B$ factories, its effects are unclear for long high-intensity bunches, as the SNS beam; hence, we investigated the mechanism of clearing of electrons with weak solenoid in this ring. The electrode-clearing system serves as a very important alternative in the SNS wherein magnets and other devices occupy most of the space. Its effects have been explored experimentally since 1992 at the PSR [20-22], and although the findings were interesting, the mechanism could not be explained.

Electrons generated by gas ionization are trapped inside the coasting beam and can cause transverse instabilities [2]. Clearing electrodes were used to clear these trapped electrons [23,24]. The clearing field pulls the 


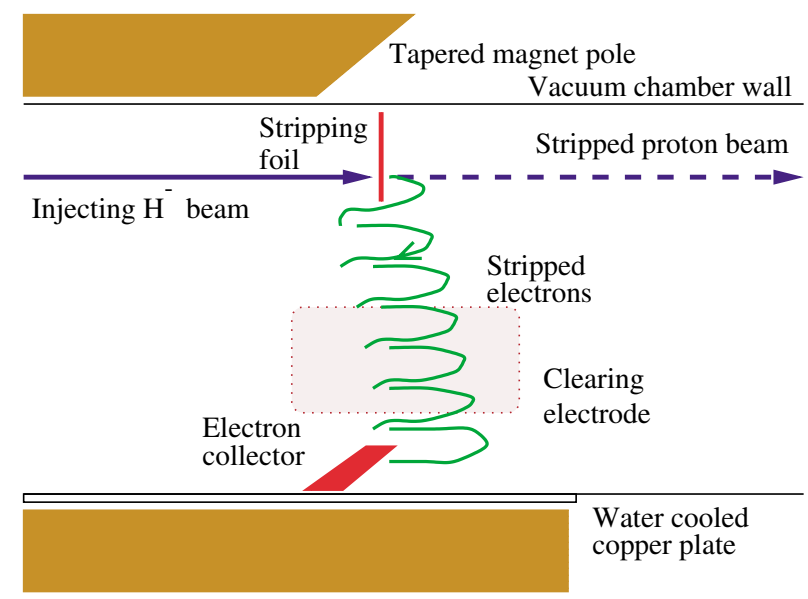

FIG. 1. (Color) Collection of stripped electrons during the injection of the $\mathrm{H}^{-}$beam at the SNS ring.

electrons out from the beam's potential well. If there is no clearing field, the electrons can be permanently trapped. On the other hand, the SNS's beam is a bunched one and most of the electrons are produced by electron multipacting. The clearing field is mainly used to suppress multipacting instead of pulling electrons out of the beam's potential well, and, at the same time, to clean the bunch gap.

One of the main sources of electrons is those generated at the stripping foil in the injection region of the SNS ring. Multiturn charge-exchange injection often is preferred for high-intensity rings to enhance the phase-space density of the accumulated beam. Near the injection stripping foil, we expect a high concentration of electrons with a broad energy spectrum. With an $\mathrm{H}^{-}$beam, the stripped electrons carry twice the current of the injecting $\mathrm{H}^{-}$beam with a kinetic energy of $m c^{2}(\gamma-1)$, where $\gamma$ is the relativistic factor of the $\mathrm{H}^{-}$beam. The injecting and circulating beams impacting on the foil produce a secondary emission of electrons at low energy (tens of eV). Both beams also generate knockon electrons at a high energy (up to several MeV). Figure 1 illustrates the collection of stripped electrons at the SNS accumulator ring. The electrons are guided by a magnetic field and collected by a water-cooled device of heat-resistant material. The electron collector consists of carbon material attached to a water-cooled copper plate. Its inner surfaces are coated with $100 \mathrm{~nm}$ thick TiN. An installed clearing electrode can apply $10000 \mathrm{~V}$. The clearing efficiency for electrons with energy up to several $\mathrm{MeV}$ still is unknown, and the combined effect of the guiding magnetic field and the clearing field also needs to be considered. A study of the clearing effect at the injection region is underway.

At the SNS accumulator ring, the BPMs (beam position monitors) around the ring were designed as clearing electrodes that can apply a voltage of up to $\pm 1000 \mathrm{~V}$ (see Fig. 2). It is not enough to completely suppress the electron cloud. However, our study shows that such a

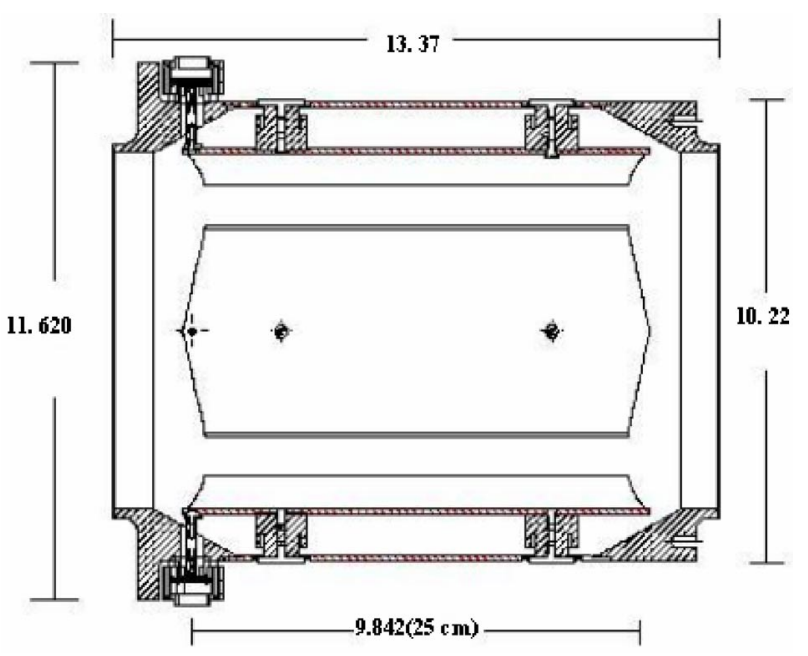

FIG. 2. (Color) Schematics of the floating-ground BPM designed for the SNS accumulator ring. A voltage of about $1000 \mathrm{~V}$ can be applied to clear the electron cloud.

voltage is effective with the "half multipacting frequency" mechanism.

The phenomenon and mechanism discussed in this paper is based upon a simulation. This paper is organized as follows. Section II describes the parameters and physical model of the simulation. Section III covers electron multipacting with clearing electrodes. In particular, we discuss a multipacting enhancement mechanism caused by the clearing electric field operating at an intermediate range. Section IV describes the clearing effect of a weak solenoid. Conclusions are given in Sec. V.

\section{SIMULATION PARAMETERS AND PHYSICAL MODEL}

The SNS beam is assumed to be cylindrical and uniformly distributed in the transverse plane. Table I gives the parameters used for the beam and machine in the simulation.

Protons incident on the chamber's inner surfaces produce primary electrons. Depending on the protons' energy and the incident angle, the yield of electrons can greatly exceed unity when the incident protons are nearly parallel to the surface [25]. In Table I, a uniform rate of

TABLE I. Simulation parameters for the SNS.

\begin{tabular}{lcc}
\hline \hline Parameter & Description & SNS \\
$E(\mathrm{GeV})$ & Beam energy & 1.9 \\
$C(\mathrm{~m})$ & Circumference & 248 \\
$N_{p}$ & Bunch population & $2.05 \times 10^{14}$ \\
$a_{x}, a_{y}(\mathrm{~mm})$ & Transverse beam size & 28,28 \\
$\tau_{b}(\mathrm{~ns})$ & Bunch length & 700 \\
$b(\mathrm{~cm})$ & Beam pipe radius & 10 \\
$P_{l}$ & Proton loss rate/turn & $1.1 \times 10^{-6}$ \\
$Y$ & Assumed proton-electron yield & 100 \\
\hline \hline
\end{tabular}


proton loss along the ring (which is far from true in the real machine), and a proton-electron yield of 100 were assumed based on comparing the simulation and experimental data from the PSR. When proton-induced electrons hit the vacuum chamber, three kinds of secondary electrons are emitted: backscattered electrons, rediffused electrons, and true secondary electrons [26-29]. The secondary-electron emission yield (SEY) is defined as a fraction of the number of electrons emitted from the metal surface to the total number of incident electrons. When SEY is larger than unity, the number of electrons increases exponentially. This phenomenon is called multipacting. The yield of backscattered electrons with zero incident angles is

$$
\delta_{e}\left(E_{0}\right)=P_{1, e}(\infty)+\left[\hat{P}_{1, e}-P_{1, e}(\infty)\right] e^{-\left(\left|E_{0}-\hat{E}_{e}\right| / W\right)^{p} / p},
$$

where $E_{0}$ is the energy of the incident electron, and other parameters are a constant value obtained by fitting the experimental results for each material. Recent experiments indicate that elastic-reflection probability approaches 1 in the limit of zero incident electron energy [30]. Therefore, the reflected electron plays a major role in SEY at low energy.

The yield of rediffused electrons with zero incident angles is

$$
\delta_{r}\left(E_{0}\right)=P_{1, r}(\infty)\left[1-e^{-\left(E_{0} / E_{r}\right)^{r}}\right],
$$

and the yield of the true secondary electrons with zero incident angles is

$$
\delta_{\mathrm{ts}}\left(E_{0}\right)=\frac{s\left(E_{0} / \hat{E}_{\mathrm{ts}}\right) \hat{\delta}_{\mathrm{ts}}}{s-1+\left(E_{0} / \hat{E}_{\mathrm{ts}}\right)^{s}} .
$$

The variables in Eqs. (1)-(3) depend on the material property of the chamber's surface. Figure 3 shows the main parameters of SEY, and Table II lists the secondary

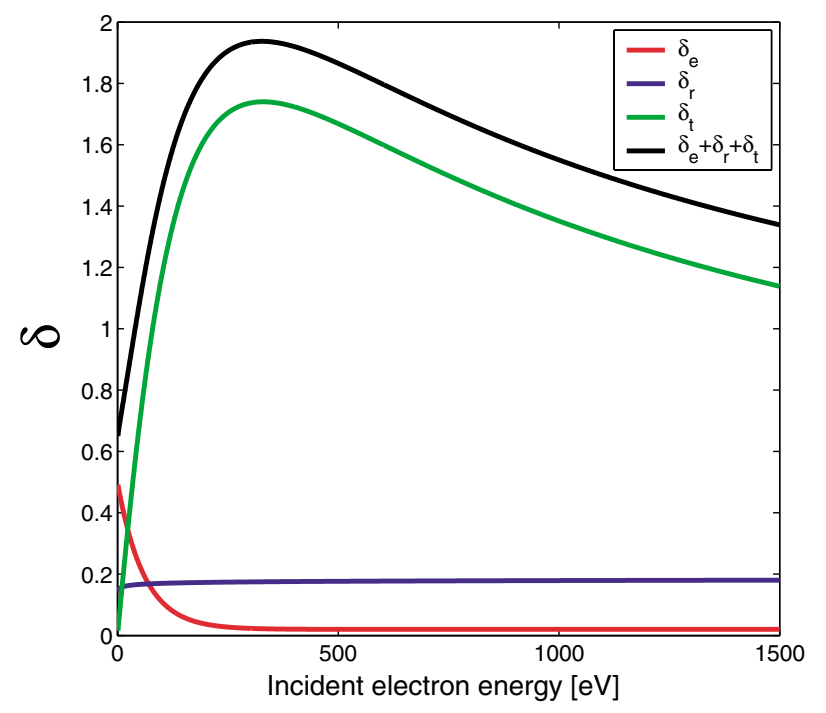

FIG. 3. (Color) Secondary emission yield.
TABLE II. Main parameters of SEY.

\begin{tabular}{cc}
\hline \hline Backscattered electrons & \\
$P_{1, e}(\infty)$ & 0.02 \\
$\hat{P}_{1, e}$ & 0.5 \\
$\hat{E}_{e}(\mathrm{eV})$ & 0 \\
$W(\mathrm{eV})$ & 60 \\
$P$ & 1 \\
Rediffused electrons & \\
$P_{1, r}(\infty)$ & 0.19 \\
$\hat{E}_{r}(\mathrm{eV})$ & 0.041 \\
$R$ & 0.104 \\
True secondary electrons & \\
$\hat{E}_{t s}(\mathrm{eV})$ & 330 \\
$\hat{\delta}_{t s}$ & 1.74 \\
$s$ & 1.526 \\
\hline \hline
\end{tabular}

emission parameters used for the simulation. The true secondary parameters are based on an experimental result [31].

The simulation program we employed is a threedimensional particle in cell code, CLOUDLAND [32]. It includes the three-dimension electron space-charge, beam-electron interaction, and various magnetic and electric fields. Primary electrons, emitted when the lost proton hits the wall, move under their own charge and the beam's space-charge and external fields. When a primary electron hits the vacuum chamber's surface, it generates secondary electrons. A statistic distribution generator controls the SEY, energy, and emission angle, obeying the experimental results. Similarly, the secondary electrons may produce tertiary electrons. Because the SEY strongly depends on the energy of the incident electrons, multipacting is closely tied to electron motion.

\section{MULTIPACTING WITH A CLEARING FIELD}

To approximate the real distribution of the SNS beam, we assume that the beam is cylindrical with a uniform transverse section. Then, the transverse space-charge fields are

$$
E_{r}(t)= \begin{cases}\frac{\lambda(t)}{4 \pi \varepsilon_{0}} \frac{2}{r} & (r>a), \\ \frac{\lambda(t)}{4 \pi \varepsilon_{0}} \frac{2 r}{a^{2}} & (r<a),\end{cases}
$$

where $\mu_{0}=4 \pi \times 10^{-7} \mathrm{H} / \mathrm{m}$ is called the permeability of the vacuum, $\varepsilon_{0}=10^{-9} / 36 \pi \mathrm{F} / \mathrm{m}$ is known as the permittivity of the vacuum, $\lambda$ is the beam-line density, $a$ is the beam's transverse size, and the beam's potential, $U$, is given by

$$
U(r, t)= \begin{cases}\frac{\lambda(t)}{4 \pi \varepsilon_{0}}\left(1+2 \ln \frac{r}{a}\right) & (r>a), \\ \frac{\lambda(t)}{4 \pi \varepsilon_{0}} \frac{r^{2}}{a^{2}} & (r<a) .\end{cases}
$$

For the SNS ring, the peak beam field at the wall's surface is $5 \times 10^{4} \mathrm{~V} / \mathrm{m}$ and the peak beam potential is 
about $6 \mathrm{kV}$. Do we need such a high clearing field or potential to clearing the electron cloud? To answer this question, we must understand the multipacting mechanism in the SNS ring. Electrons initially are emitted with low energy when the lost protons hit the chamber's wall surface. In the SNS ring, where "trailing-edge multipacting" is dominant, electrons generated after the peak of the beam pulse will be accelerated towards the center of the beam and decelerated after passing through it. These electrons will gain energy as they reach the opposite wall. If the gain is high enough, the SEY can exceed unity, thereby amplifying electron density with successive traversals across the beam pipe. The electron energy, when one electron drifts from one side of the chamber's wall to the opposite surface and hits it, can be estimated as [33]

$$
E_{\text {strike }}=-\frac{1}{2} \sqrt{\frac{m e}{2 \pi \varepsilon_{0}}} \beta c\left(a(2 \zeta-1) \arcsin \frac{1}{\sqrt{\zeta}}+a \sqrt{2 \ln \frac{b}{a}}+\sqrt{2} \zeta \int_{a}^{b} \frac{d r}{\sqrt{\ln (b / r)}}-\frac{1}{\sqrt{2}} \int_{a}^{b} \frac{1+2 \ln (r / a)}{\sqrt{\ln (b / r)}} d r\right) \frac{\partial \lambda}{\partial z} \frac{1}{\sqrt{\lambda}}
$$

with $\zeta=1+2 \ln (b / a)$, where $b$ is the chamber's radius, $m$ and $e$ are the mass and the charge of the electron, respectively. Figure 4 shows the electron's energy when it strikes on the chamber wall's surface based on Eq. (6) and the program CLOUDLAND. The SEY also is depicted; it shows that multipacting starts around 500 ns. Strong multipacting occurs in the tail of the bunch due to the high electron energy there. The electron energy given by Eq. (6) can clearly explain the mechanism of trailingedge multipacting.

A clearing system was applied to the SNS injection area and the BPMs were modified as clearing electrodes. We assumed a clearing electrode with a vertically uniform field in this study. The clearing field is equal to the total voltage between clearing electrodes divided by the chamber's diameter. In principle, to suppress the electron cloud, a clearing field is required equal to the maximum beam space-charge field at the wall's surface to restrain the emission of the secondary electrons. An adequate clearing field should be applied to suppress the emission of secondary electrons at the bunch tail where

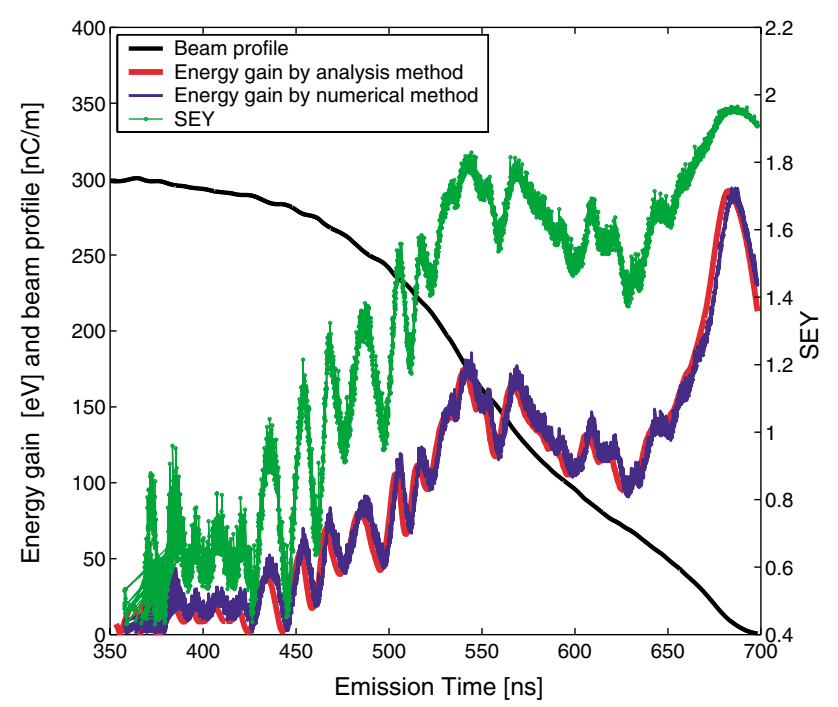

FIG. 4. (Color) Comparison of energy gain. The blue line is the energy evaluated numerically, the red line is the energy value obtained with Eq. (6), the black line is the beam's profile, and the green line is the estimation of the SEY. multipacting occurs. Multipacting depends on many parameters, such as bunch intensity, the shape of the longitudinal beam's profile, and the size of the transverse beam's profile. We can estimate the electrons' energy gain using Eq. (6) to find the starting time of multipacting where the total SEY exceeds unity, and then calculate the beam's space-charge field near the chamber wall's surface at that moment. This space-charge field is the required electric clearing field to suppress multipacting. For example, at the SNS, a clearing voltage of $8 \mathrm{kV}$ is needed to complete suppress multipacting after $500 \mathrm{~ns}$. In fact, the requirement on the clearing field is not directly related to the beam's potential because the electrons produced by multipacting at the bunch tail are emitted at the wall's surface and they could not be trapped by the beam's potential, even without any clearing field [33]. The purpose of the clearing field is to suppress the emission of secondary electrons instead of pulling them out from the beam's potential like trapped ions or electrons. On the other hand, a necessary condition for removing trapped electrons from a coasting proton beam or ions created by residual gas ionization in an electron machine is that the clearing electrode's electric field should be higher than the maximum field generated by the beam's space charge because the beams can deeply trap electrons and ions at the chamber's center.

To find the correct clearing field, various clearing voltages were applied, and their effects were simulated using ClOUdLAND. Figure 5 shows the buildup of the electron cloud during the passage of one bunch at various clearing voltages. As a figure of merit, we use the peak line electron density to describe the clearing field's efficiency. As Fig. 6 shows, a notable feature is that this efficiency is not a monotonic function of the clearing voltage. A weak clearing field of $200 \mathrm{~V}$ reduces the line density of the electron by about a factor of 3 . Subsequently, the line density increases with the increasing voltage, reaching a maximum at $2000 \mathrm{~V}$; it decreases again when stronger clearing fields are applied.

It is not surprising that a $6 \mathrm{kV}$ clearing voltage, which equals the beam's peak potential for the SNS ring, can suppress most of the multipacting. We expect $8 \mathrm{kV}$ to complete to suppress it, as discussed above. In agreement with our estimation, we note that the peak potential 


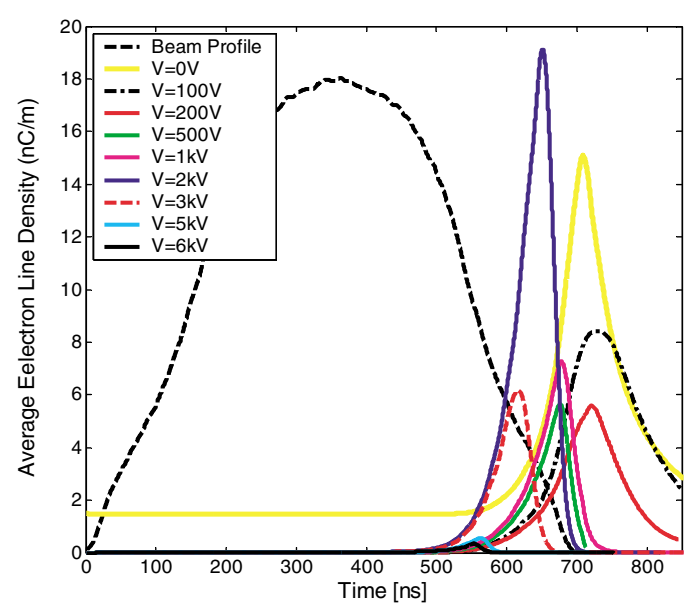

(a)

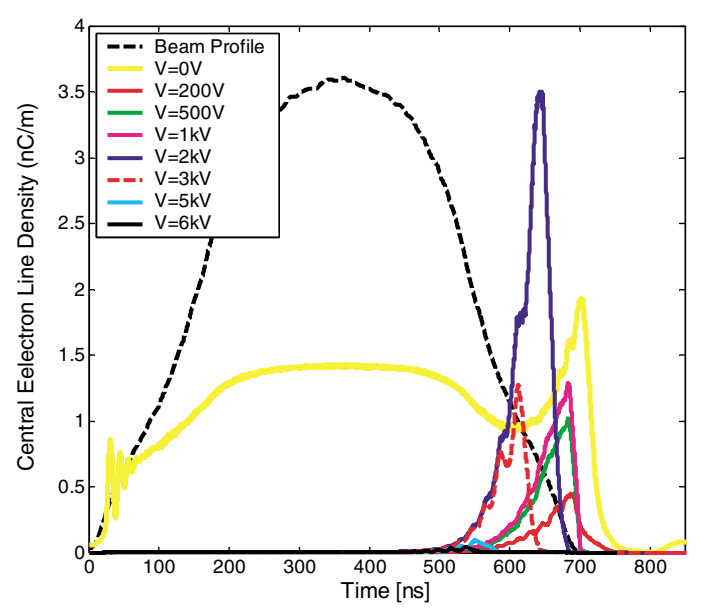

(b)

FIG. 5. (Color) Electron cloud buildup with various clearing voltages in the SNS's drift region. (a) The electron cloud line density inside the vacuum chamber, and (b) inside the beam.

of $6 \mathrm{kV}$ is not necessary for clearing the electron cloud (Fig. 6). Unexpectedly, however, we found that the clearing efficiency is higher for $200 \mathrm{~V}$ than for $3000 \mathrm{~V}$, and that multipacting is stronger with a $2000 \mathrm{~V}$ clearing field than any other one.

The electron motion in a clearing field, shown in Fig. 7, can explain these results. Electron motion can be divided into two categories: electrons bouncing between the chamber walls' surfaces with a low clearing field and bouncing near the positive clearing electrode with a high clearing field.

Without any clearing field, an electron at the bunch tail bounces from one side of the walls' surface to the opposite side and produces secondary electrons that then will rebound. In this case, the electron's energy at the walls' surface ranges from 0 to $300 \mathrm{eV}$. When a low clearing voltage is applied, for example, $200 \mathrm{~V}$, the electron still bounces between the walls' surfaces. However, the clearing field can effectively reduce the electrons' energy at the surface closer to the negative electrode and increase it

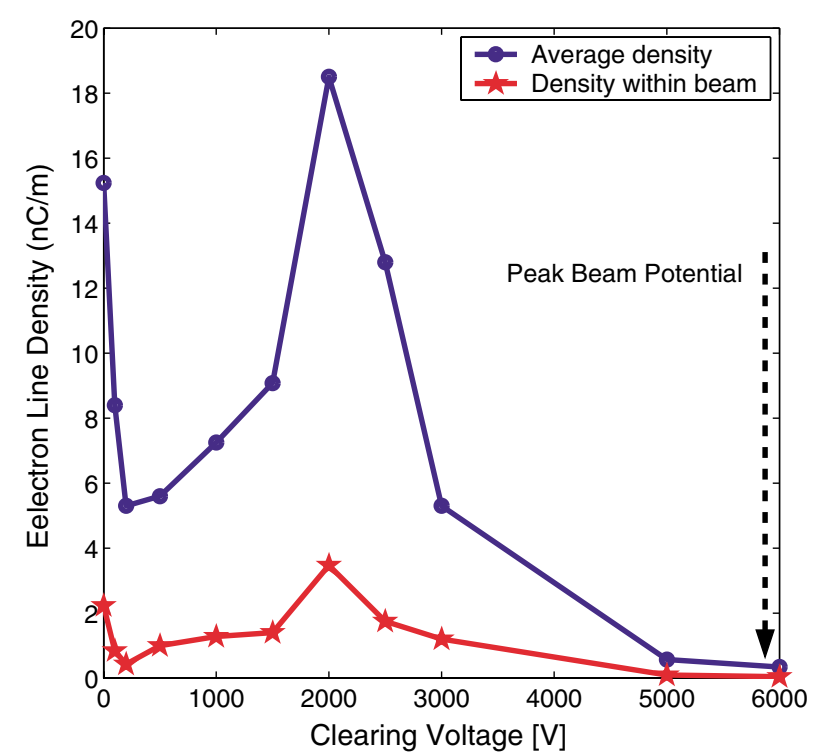

FIG. 6. (Color) Variation of the peak line density of the electron cloud with various clearing potentials in the SNS's drift region.

at the surface closer to the positive electrode. As a result, the yield of secondary emissions at the latter surface increases while decreasing near the negative electrode due to the relationship between the energy of the incident electron and the SEY (Fig. 3). Therefore, the electron density can be reduced effectively, although multipacting still happens at the surface near the positive electrode. This "alternate multipacting" mechanism ensures that a low clearing field can efficiently lower electron density. The clearing voltage working with an alternate multipacting mechanism should be smaller than the peak of electron-energy gain in the zero field case divided by the electron charge $e$, which is $300 \mathrm{~V}$ for SNS's ring.

When the clearing field increases, for example, to $500 \mathrm{~V}$ (Fig. 7), the electron cannot reach the walls' surface near the negative electrode. Instead, the clearing field turns it back inside the chamber, so the electron reaches the surface closer to the positive electrode than to the location of its genesis, until it finally reaches the electrode itself and moves along the vertical lines of the clearing field. This process is quite similar to "clearing field polarization." Without a clearing field, electrons mainly move radially and have uniform azimuthal distribution. The clearing field can change the electron's orbit. The destination of every electron's orbit is the clearing field line. We say that the electrons are finally polarized by the clearing electric field when they move along the clearing field lines. The "polarization time" depends on the strength of the clearing field: the stronger the field, the shorter the polarization time.

When an electron bounces only from one side of the chamber's surface, the frequency at which it hits the walls' surface will be reduced by about half, and hence, 

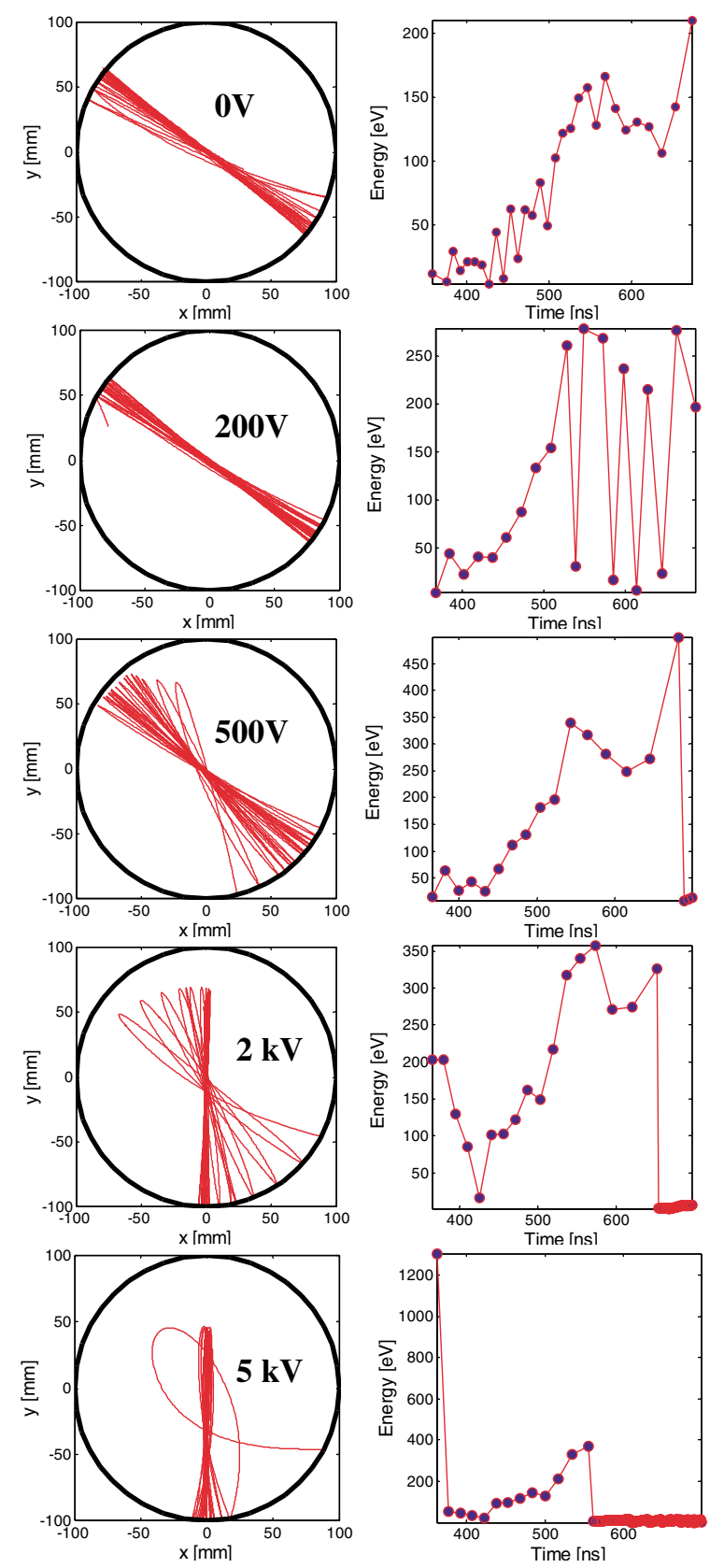

FIG. 7. (Color) Examples of an electron's orbit (left column) in the transverse plane and its energy at the walls' surface (right column) with various clearing voltages. The red lines in the left column denote the orbits, and the solid black lines show the vacuum chamber's shape.

the density of the electron cloud could be less than when there is no clearing field. The effect of half multipacting frequency can explain the result with the $500 \mathrm{~V}$ field. We note that the electron density with the $200 \mathrm{~V}$ clearing voltage is smaller than that with $500 \mathrm{~V}$ (Fig. 6), because the alternate multipacting (the former) results in a lower density compared with the effect of the half multipacting frequency (the latter). For example, we assume a SEY of 1.8 and 0.7 for the multipacting and no-multipacting cases, respectively. The alternate multipacting gives an effective SEY of 1.26 for once multipacting and no multipacting. In the case of the half multipacting frequency, the chance of multipacting is reduced to half, but the SEY is the same as the multipacting SEY 1.8. Consequently, the half multipacting frequency gives an effective SEY of 1.8 that is larger than the effective SEY in alternate multipacting, 1.26.

By further increasing the clearing field, for example, to $2000 \mathrm{~V}$ (see Fig. 7), the multipacting starts earlier around the beam's pulse peak due to the gain in the electron's energy from the clearing field; by contrast, in a low or zero clearing field, multipacting could happen only at the tail of the beam pulse (Fig. 4). As a result, there may be stronger multipacting in the presence of a suitable clearing field than in its absence.

The clearing field's effect on the electron's energy when it hits the wall's surface strongly depends on the electron's orbit because the energy received from the clearing field corresponds to the difference in the clearing field's potential between its emission and striking points. If the clearing field is weak, for example, $500 \mathrm{~V}$, the polarization time is long (which is more than $350 \mathrm{~ns}$ in the example of Fig. 7), and hence, when the field forces back the electron, the location where it strikes the wall is close to its emission point. Therefore, a weak clearing field has a weak effect on the electron's energy at the wall surface during "polarization." However, with a stronger clearing field, the polarization time is shorter and the electron can gather more energy from the clearing field during this process. For example, in Fig. 7, the polarization times are 120 and $50 \mathrm{~ns}$ for 2000 and $5000 \mathrm{~V}$ cases, respectively. After the electron is polarized, it can no longer receive energy from the clearing field because it will strike on the same position of the chamber surface or electrodes. A stronger clearing field generates higher electron energy at the surface but a shorter polarization time, and hence, a short extra multipacting time due to the clearing fields. Therefore, an unsuitable clearing field, $2000 \mathrm{~V}$, can maximize electron multipacting.

An extreme clearing field, for example, $5000 \mathrm{~V}$, has a weak effect on the electrons' energy at the walls' surface except in a very short polarization time (about $50 \mathrm{~ns}$ ), and it can effectively suppress the emission of secondary electrons at the bunch tail where the clearing field could be stronger than the beam's space-charge field at the wall surface. Therefore, a strong clearing field can effectively reduce the electron cloud's density by reducing the chance of multipacting at the bunch's tail, with a weak effect at the bunch's center. A clearing field equal to the maximum beam's space-charge field at the walls' surface will completely suppress multipacting.

Figures 8 and 9 show the transverse distribution of the electron cloud at different times for 0 and $2000 \mathrm{~V}$ clearing voltage. The distribution is azimuthally uniform at zero 


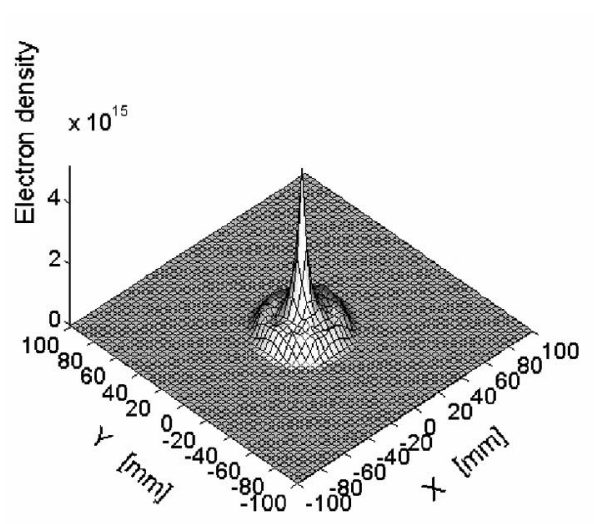

(a)

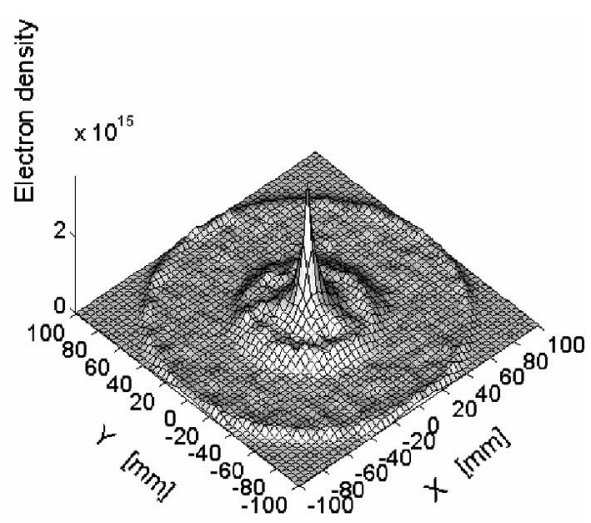

(b)

FIG. 8. Electron distribution in the transverse plane at (a) $350 \mathrm{~ns}$ and (b) $630 \mathrm{~ns}$ with zero clearing voltage.

fields. However, the electron cloud is distributed along the line of the clearing field (vertical here) at the horizontal center due to the "polarization effect" of the clearing field.

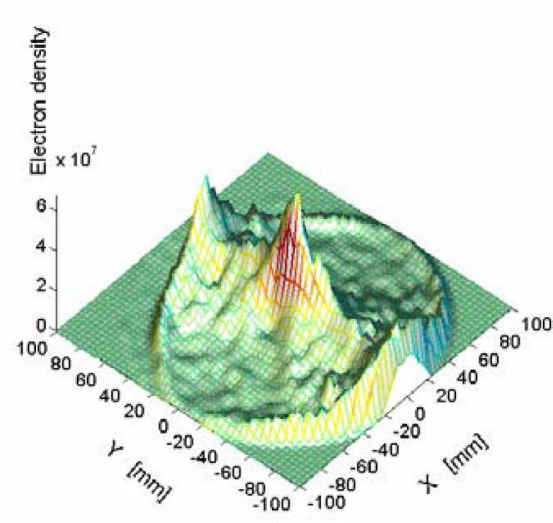

(a)

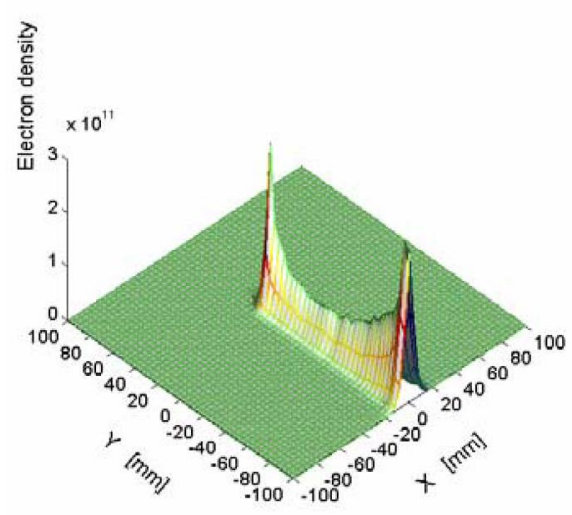

(c)
Inside strong dipole magnets, strong multipacting happens at the horizontal chamber center [33]. A horizontal electric field can longitudinally pull the electrons out of the magnet to drift region by the crossed-field drift and

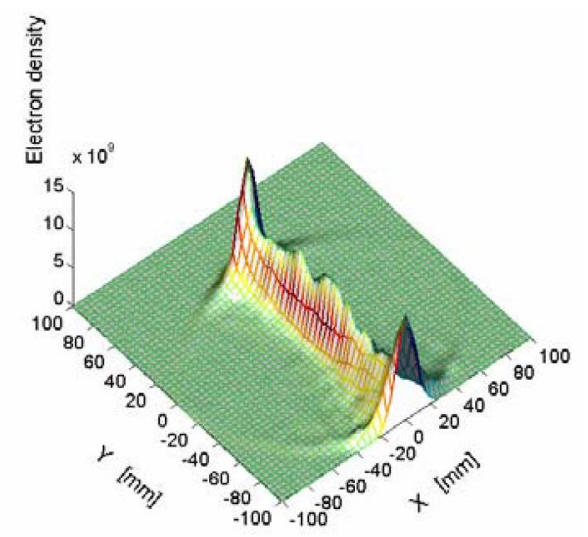

(b)

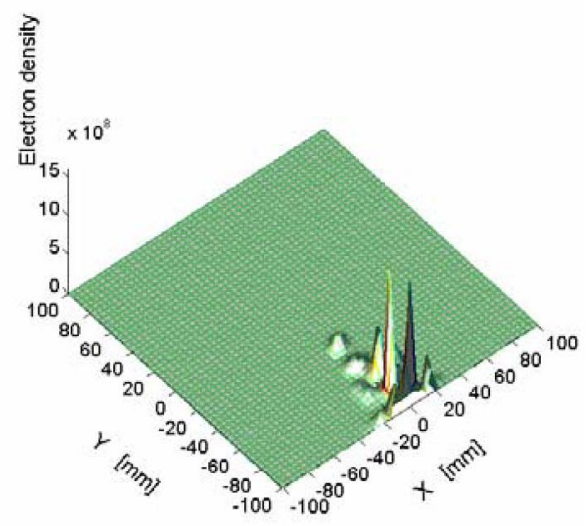

(d)

FIG. 9. (Color) Electron distribution in the transverse plane with $2000 \mathrm{~V}$ clearing potentials at different times (a) $350 \mathrm{~ns}$, (b) $560 \mathrm{~ns}$, (c) $630 \mathrm{~ns}$, and (d) $700 \mathrm{~ns}$. 


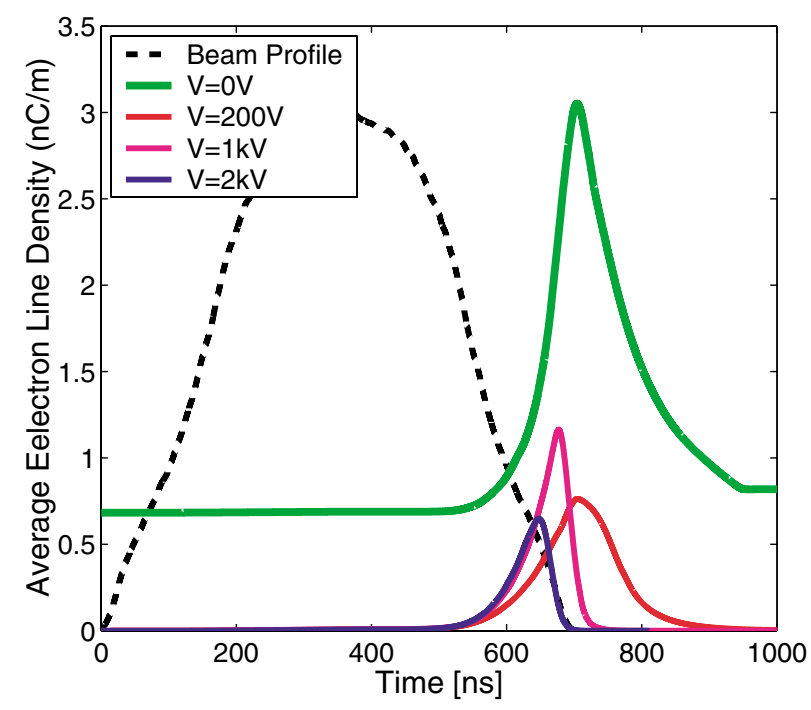

FIG. 10. (Color) Electron cloud buildup with various clearing voltages in the SNS's dipole magnet.

then a solenoid or electrode can be applied to clear them. However, this requires a strong horizontal electric field to pull them out of the strong dipole magnet within the multipacting period because the effects are inversely proportional to the magnetic field's strength. For example, it requires an electric field of $1.4 \mathrm{MV} / \mathrm{m}$ to pull out electrons from a $1.0 \mathrm{~T}$ dipole magnet with a length of $0.5 \mathrm{~m}$ within a half bunch pulse time of $350 \mathrm{~ns}$. Therefore, to effectively repel electrons, the clearing electric field must be applied along the lines of the magnetic field. This statement is true for other strong magnetic fields. To excite an effective vertical clearing field in a dipole magnet, a system similar to the one in the drift region must be applied. Figure 10 shows such a clearing effect in a dipole magnet. The clearing field always has a positive effect, in contrast to its effect in the drift region, because the electron can only effectively move along the strong magnetic field lines. Therefore, there is no "polarization process." The important feature is that the "alternating multipacting" mechanism makes a weak clearing field of $200 \mathrm{~V}$ more effective than $1000 \mathrm{~V}$.
In quadrupole and sextuple magnets, very weak multipacting occurs around the middle of each magnetic pole surface because only those electrons moving along those field lines receive enough energy. The simulated electron cloud is more than 2 orders of magnitude smaller than in the drift region due to the low electron energy at the wall's surface. Therefore, a clearing electrode is not necessary in the quadrupole magnet due to the absence of multipacting. However, a strong electron signal was observed in LANL-PSR's quadrupole magnet and one experiment study is planned to measure the electron cloud in quadrupole [34]. Further detailed studies should be done to solve the discrepancy between our simulation and PSR's observation.

\section{CLEARING AN ELECTRON CLOUD WITH SOLENOIDS}

In a short bunch machine, the electron's orbit is close to a half circle if the bunch gap is long enough. Therefore, a weak solenoid with a gyration period less than half a bunch gap can efficiently confine electrons near the wall surface of the vacuum chamber. The required strength of the solenoid field can be estimated using the criterion

$$
\rho \ll a,
$$

where $\rho$ is the Larmor radius

$$
\rho=\frac{\sqrt{2 E m_{e}}}{e B}
$$

where $E$ is the electron energy.

For example, in the KEKB, the electron energy around the wall's surface is limited by the energy received from the positron bunch; presently, it is about $50 \mathrm{eV}$. The Larmor radius is $5 \mathrm{~mm}$ with a $50 \mathrm{G}$ solenoid field for a $50 \mathrm{eV}$ electron.

The peak electron energy at the wall is $300 \mathrm{eV}$ without a magnetic field in the SNS ring (Fig. 4). Equation (8) gives a Larmor radius of $20 \mathrm{~mm}$ for $300 \mathrm{eV}$ electron in a $30 \mathrm{G}$ field. However, simulation shows that the maximum Larmor radius is much larger (Fig. 11). In fact, Eq. (8) applies only for short bunches where there is no beam
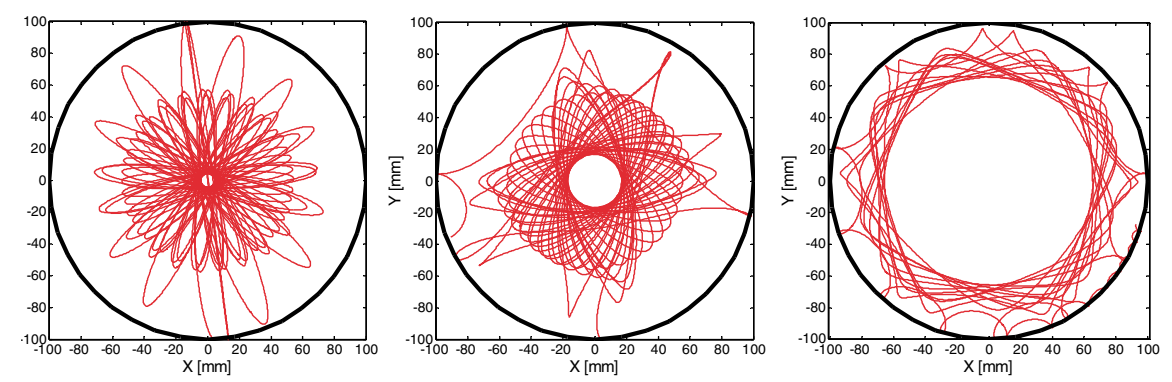

FIG. 11. (Color) Transverse electron trajectories in different solenoid fields (left panel) 0 G, (middle panel) $10 \mathrm{G}$, and (right panel) $30 \mathrm{G}$. 

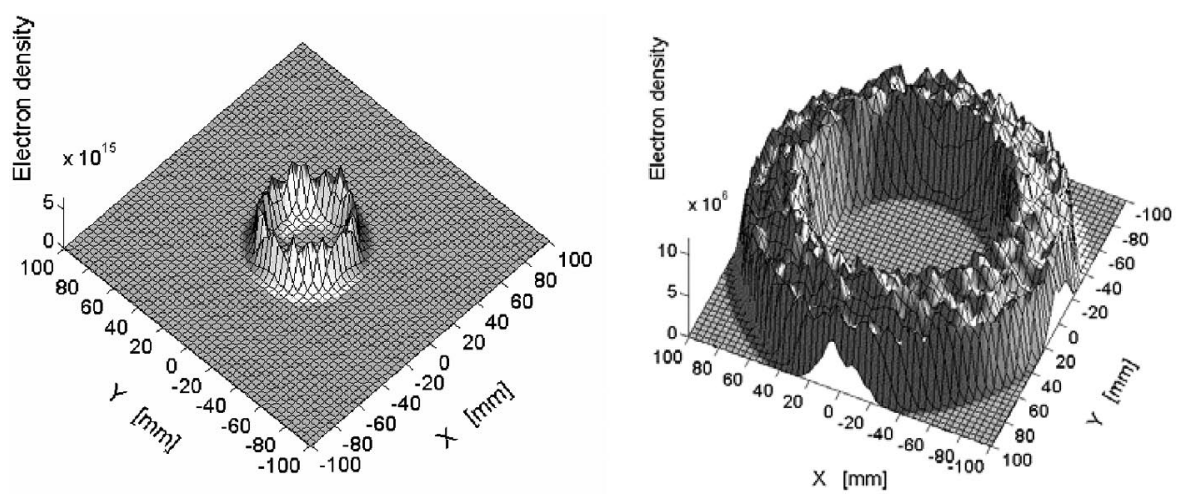

FIG. 12. Electron cloud's transverse distribution in a $10 \mathrm{G}$ (left panel) and $30 \mathrm{G}$ (right panel) solenoid field during passage through the bunch center.

space charge when an electron gyrates during the bunch gap. There is a different clearing mechanism for long bunches where the force of the beam's space charge plays an important role in confining the electrons. The transverse motion of an electron in a polar coordinate system is given by

$$
\begin{aligned}
& m\left(\frac{d^{2} r}{d t^{2}}-r \frac{d \phi}{d t} \frac{d \phi}{d t}\right)=e v_{\phi} B_{z}+e E_{r}, \\
& m\left(\frac{d^{2} \phi}{d t^{2}}+2 \frac{d r}{d t} \frac{d \phi}{d t}\right)=e v_{r} B_{z} .
\end{aligned}
$$

When the solenoid-field's force is weaker than the beam's space-charge force, below $30 \mathrm{G}$ for the SNS ring, electrons move around the beam's center. The combination of the transverse beam's space-charge field and the longitudinal solenoid field causes the electron to make elliptical-like motions with an azimuthal rotation due to the cross-field drift. Otherwise, when the solenoid-field force is dominant, the electron moves near the wall surface with a half circlelike orbit. The radius of gyration can be roughly estimated using

$$
\left\{\begin{array}{l}
\rho=\frac{\sqrt{2 E(b-\rho) m_{e}}}{e B}, \quad(r>a) . \\
E(r)=\frac{\lambda_{\text {peak }}}{2 \pi \varepsilon_{0}} \ln \frac{b}{4},
\end{array}\right.
$$

This estimation includes the peak beam-line density because its peak potential causes the largest radius. The estimated $\rho$ is 18 and $5 \mathrm{~mm}$ for 60 and $100 \mathrm{G}$ solenoid fields, respectively.

Figure 11 shows sample orbits in different solenoid fields. The orbits depicted can explain the electron cloud's distribution in a solenoid field (Fig. 12). Figure 13 shows the electron peak density with different solenoid fields; it decreases exponentially with the increment of solenoidfield strength. Simulation shows that a $30 \mathrm{G}$ field can significantly reduce the density of the electron cloud by a factor of 1000, and a $40 \mathrm{G}$ field completely suppresses multipacting. Most importantly, a $30 \mathrm{G}$ solenoid can confine the electrons near the walls' surface without allowing any inside the beam. Therefore, a solenoid can efficiently improve the beam's stability and suppress multipacting because an electron cannot derive sufficient energy from the beam's potential.

In the above simulation, uniform solenoid fields were assumed. However, the real solenoid field must be discussed because most of the ring is occupied by magnet and other devices. When the periodic solenoids are arranged with the same current direction in the coil, this geometry is called equal polarity configuration. The magnetic field can be exactly expressed as an expansion of series [35] as

$$
B_{r}=B_{0} \frac{2 k d}{\pi} \sum_{n=1}^{\infty} \sin n h k K_{1}(n k d) I_{1}(n k r) \sin n k z
$$

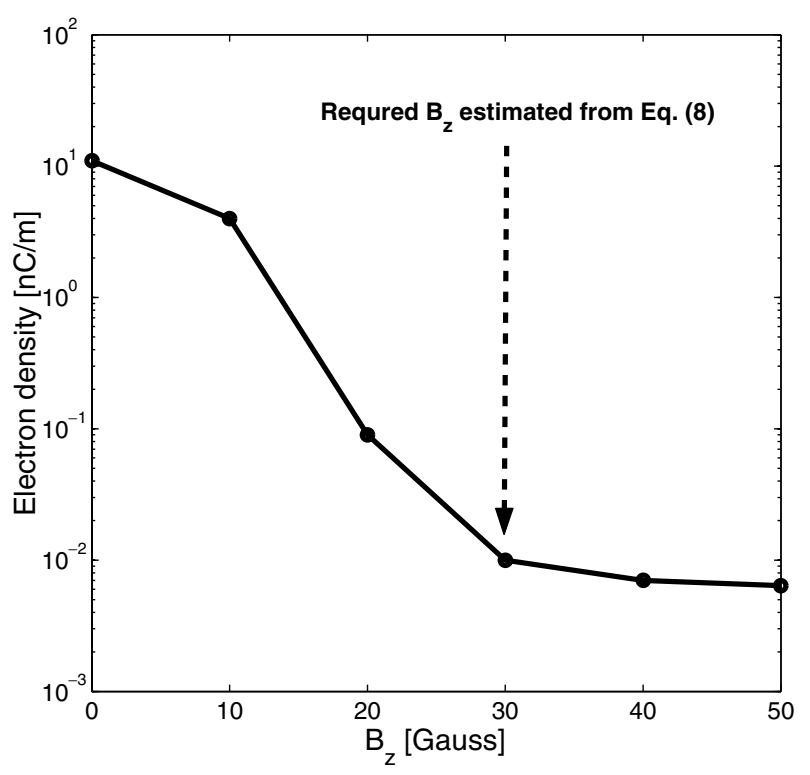

FIG. 13. Reduction of electron line density with increasing uniform solenoid fields. The dashed line indicates the required field estimated from Eq. (8) with $\rho$ of $20 \mathrm{~mm}$ and energy $300 \mathrm{eV}$. 


$$
B_{z}=B_{0}\left(\frac{2 h}{\lambda}+\frac{2 k d}{\pi} \sum_{n=1}^{\infty} \sin n h k K_{1}(n k d) I_{0}(n k r) \cos n k z\right)
$$

where the $K_{n}$ and $I_{n}$ are modified Bessel functions of the order $n, d$ is the solenoid's radius, $2 h$ the solenoid's length, $k=2 \pi / \lambda$, and $\lambda$ is the distance between adjacent solenoids with equal polarity.

When the solenoid's current takes an alternating direction, it is called an opposite polarity configuration. There is a similar expression for this configuration:

$$
\begin{aligned}
& B_{r}=B_{0} \frac{4 k d}{\pi} \sum_{n=1,3,5}^{\infty} \sin n h k K_{1}(n k d) I_{1}(n k r) \sin n k z \\
& B_{z}=B_{0} \frac{4 k d}{\pi} \sum_{n=1,3,5}^{\infty} \sin n h k K_{1}(n k d) I_{0}(n k r) \cos n k z
\end{aligned}
$$

Figure 14 shows the effects of solenoid configuration on electron cloud buildup and distribution, with $B_{0}=50 \mathrm{G}$, $h=0.4 \mathrm{~m}, d=120 \mathrm{~mm}, \lambda=1$ and $2 \mathrm{~m}$ for the equal and the opposite polarity cases, respectively. The electron density in the latter case is 6 times larger than that in the former case. Importantly, most electrons stay around the chamber's center in the opposite polarity case; there are no electrons near the chamber's center with an equal polarity configuration. Therefore, the solenoid should be arranged in the latter configuration in operating the real machine. The same conclusion was reached for $B$ factories [36,37]. Simulation shows that the electron cloud in an opposite configuration is trapped inside the solenoids rather than in the gap between the solenoids. The distribution reflects the combined effect of the beam's spacecharge force and the solenoid fields.

With short bunches, such as $B$ factories, resonance occurs when the time that an electron emerges from the wall and is bent back by the magnetic field coincides with the interval between two consecutive bunches $[38,39]$

$$
T / 2=S_{b} / c
$$

where $T$ is the period of gyration motion $2 \pi m_{e} / e B$. This period is $7.2 \mathrm{~ns}$ for a $50 \mathrm{G}$ field. The required solenoid field

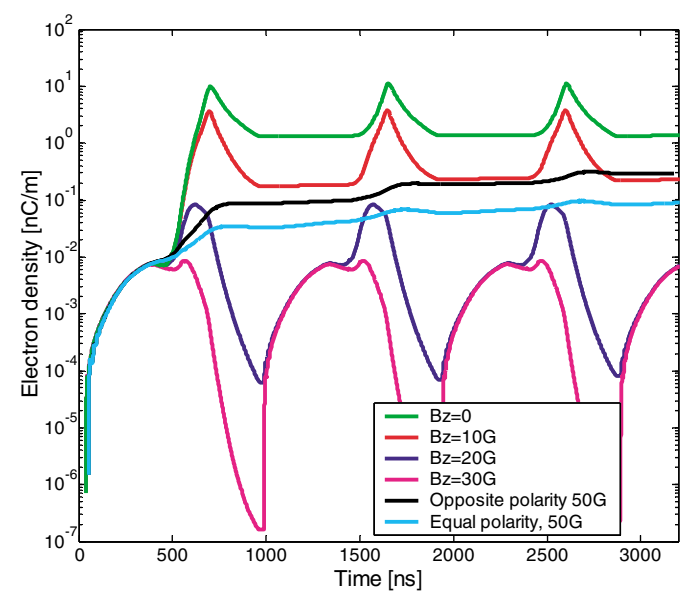

(a)

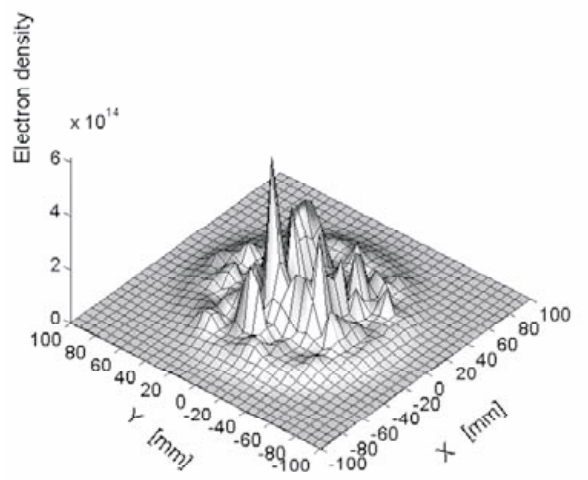

(b)

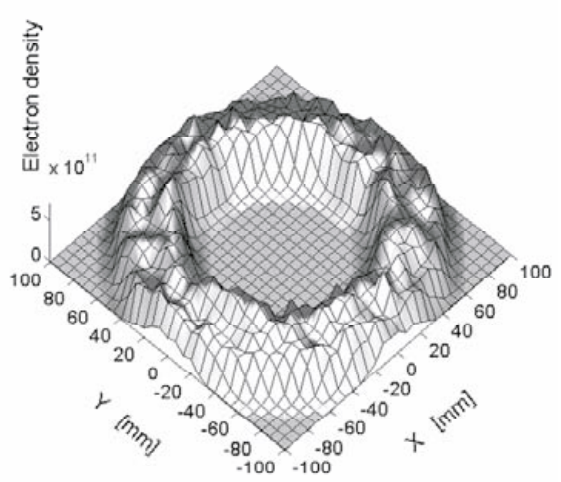

(c)

FIG. 14. (Color) Effects of solenoid's configuration on the buildup and distribution of the electron cloud. (a) Electron cloud's buildup, (b) its distribution with opposite polarity configuration, and (c) its distribution with equal polarity configuration. 
depends on the bunch's current. The typical solenoid field is about $50 \mathrm{G}$ in KEK and PEPII $B$ factories and LANL's PSR. Both the bunch length and bunch gap between subsequent bunches in the SNS ring, which are 700 and $300 \mathrm{~ns}$, respectively, are much longer than the electron's gyration period in the clearing solenoid fields. As a result, Eq. (15) could not be satisfied for a long bunch beam. Therefore, the SNS exhibits no such kind of resonance phenomenon as shown in Fig. 13.

Solenoids in KEKB Low Energy Ring significantly reduce the transverse beam size and improve the luminosity [8]. However, the solenoids in PSR reduce only the electron cloud signal but have no improvement on the transverse instability threshold [40]. This still is a puzzle.

\section{CONCLUSION}

We investigated the mechanism of action of a clearing field applied to electron cloud buildup for a long bunch. In the drift region, both a low clearing voltage of $200 \mathrm{~V}$ or a high clearing voltage of $5000 \mathrm{~V}$ proved efficient. However, the numerical study suggested that a clearing field may introduce strong multipacting at a intermediate voltage. For the SNS, the strongest multipacting occurs at a clearing voltage of $2000 \mathrm{~V}$. The mechanism is explained by the effect of the clearing field on the electrons' energy at the wall surface. The entire clearing phenomenon can be understand from studying electron motion. The BPMs in the SNS ring are helpful in lowering electron density at a low voltage (hundreds of $\mathrm{V}$ ) with the alternate multipacting or half multipacting frequency mechanism. On the other hand, clearing fields always have a positive effect in dipole magnet. The required clearing field is the same as in the drift region. A solenoid significantly suppresses electron multipacting without creating any resonance due to the long bunch length and bunch gap; an equal polarity configuration is much more efficient than an opposite polarity configuration. Indeed, it is a perfect remedy for suppressing multipacting with zero electron cloud density inside the beam in the drift region.

\section{ACKNOWLEDGMENTS}

We thank Y. Y. Lee, M. Blaskiewicz, P. He, A. Fedotov, and R. Macek for discussions, P. Cameron, J. Brodowski, J. Tuozzolo, and H. Hseuh for help in practically implementing the clearing electrodes, and M. Furman for supplying data for benchmarking our code.

[1] G. I. Budker, G. I. Dimov, and V. G. Dudnikov, in Proceedings of the International Symposium on Electron and Positron Storage Rings, Saclay, 1966 (Universitaiers De France, Orsay, 1966), p. VIII-6-1.

[2] H. G. Hereward, CERN Report No. 71-15, 1971.

[3] E. Keil and B. Zotter, CERN Report No. CERN-ISR-TH/ 71-58, 1971.
[4] R. J. Macek, A. Browman, D. Fitzgerald, R. C. McCrady, F. E. Merrill, M. A. Plum, T. Spickermann, T.-S. Wang, K. C. Harkay, R. Kustom, R. A. Rosenberg, J. E. Griffin, K. Y. Ng, and D. Wildman, in Proceedings of the Particle Accelerator Conference, Chicago, 2001 (IEEE, Piscataway, NJ, 2001), p. 688.

[5] M. Blaskiewicz, in Workshop on Instabilities of HighIntensity Hadron Beams in Rings, edited by T. Roser and S. Y. Zhang, AIP Conf. Proc. No. 496 (AIP, New York, 1999), p. 321.

[6] M. Izawa, Y. Sato, and T. Toyamasu, Phys. Rev. Lett. 74, 5044 (1995).

[7] K. Ohmi, Phys. Rev. Lett. 75, 1526 (1995).

[8] H. Fukuma, CERN Report No. CERN-2002-001, 2002, pp. $1-10$.

[9] Sam Heifets, in Proceedings of the 8th Advanced Beam Dynamics Mini-Workshop on Two-Stream Instabilities in Particle Accelerators and Storage Rings, Santa Fe, New Mexico, 2000, http://www.aps.anl.gov/conferences/icfa/ two-stream.html

[10] Z.Y. Guo, H. Huang, S. P. Li, D. K. Liu, L. Ma, Q. Qin, L. F. Wang, J. Q. Wang, S. H. Wang, C. Zhang, F. Zhou, Y. H. Chin, H. Fukuma, S. Hiramatsu, M. Izawa, T. Kasuga, E. Kikutani, Y. Kobayashi, S. Kurokawa, K. Ohmi, Y. Sato, Y. Suetsugu, M. Tobiyama, K. Yokoya, and X. L. Zhang, in Proceedings of the 1st Asian Particle Accelerator Conference, Tsukuba, Japan, 1998 (High Energy Accelerator Research Organization, Tsukuba, Japan, 1998), pp. 432-434.

[11] J. M. Jimenez, G. Arduini, P. Collier, G. Ferioli, B. Henrist, N. Hilleret, L. Jensen, K. Weiss, and F. Zimmermann, LHC Project Report No. 632, 2003.

[12] K. Cornlis, CERN Report No. CERN-2002-001, 2002, pp. 11-16.

[13] O. Gröbner, in Proceedings of the 10th International Conference on High Energy Accelerators, Serpukhov, 1977 (USSR Academy of Science, Moscow, 1977), p. 277.

[14] O. Gröbner, in Proceedings of the Particle Accelerator Conference (PAC97), Vancouver, 1997 (IEEE, Piscataway, NJ, 1997), p. 3589.

[15] R. Macek, PSR Technical Note No. PSR-00-10, 2000; also V. Danilov, A. Aleksandrov, J. Galambos, D. Jeon, J. Holmes, and D. Olsen, in Proceedings of the Workshop on Instabilities of High-Intensity Hadron Beams in Rings, Upton, New York, 1999, AIP Conf. Proc. No. 496 (AIP, New York, 1999), p. 315.

[16] O. Bruning, CERN LHC Project Report No. 158, 1997.

[17] F. Zimmermann, CERN SL Note No. 2001-022 (AP), 2001.

[18] L. Wang, H. Fukuma, and S. Kurokawa, "Clearing of Photoelectron Cloud in Various Magnetic Fields using Multi-Stripline Electrodes," KEK (to be published).

[19] F. Caspers, in Proceedings of ECLOUD'02, CERN, Geneva, 2002 (unpublished).

[20] M. Plum, D. Brown, R. Macek, and T. S. Wang, PSR Tech Note No. 94-03, 1994.

[21] M. Plum, J. Allen, M. J. Borden, D. H. Fitzgerald, R. J. Macek, and T. S. Wang, PSR Tech Note No. 95-02, 1995.

[22] M. Plum, PSR Tech Note No. 96-13, 1996.

[23] E. Fisher, CERN Report No. ISR-VAC/66-15, 1966. 
[24] A. G. Ruggiero, FNAL Report No. TM-714 1600, 1977.

[25] P. Thieberger, A. L. Hanson, D. B. Steski, V. Zajic, S. Y. Zhang, and H. Ludewig, Phys. Rev. A 61, 042901 (2000).

[26] M. A. Furman and M.T. F. Pivi, Phys. Rev. ST Accel. Beams 5, 124404 (2002).

[27] H. Bruining, Physics and Applications of Secondary Electron Emission (Pergamon Press, New York, 1954).

[28] P. A. Redhead, J. P. Hobson, and E.V. Kornelsen, The Physical Basis of Ultrahigh Vacuum (Chapman and Hall, London, 1968), Chap. 4 (reprinted by the AIP in 1993 as part of the American Vacuum Society Classics series).

[29] H. Seiler, J. Appl. Phys. 54, R1 (1983).

[30] R. Cimino, in Proceedings of the ICFA Mini-WorkshopWorking Group on High Luminosity $e^{+} e^{-}$Colliders, Alghero (SS), Italy, 2003, http://www.lnf.infn.it/ conference/d2/gener.htm

[31] P. He (private communication).

[32] L. F. Wang, H. Fukuma, K. Ohmi, S. Kurokawa, K. Oide, and F. Zimmermann, Phys. Rev. ST Accel. Beams 5, 124402 (2002).
[33] L. Wang, M. Blaskiewicz, J. Wei, K. Ohmi, and R. Macek, in Proceedings of the 13th ICFA Beam Dynamics MiniWorkshop Beam Induced Pressure Rise in Rings, Brookhaven National Laboratory, Upton, NY, 2003, http://www.c-ad.bnl.gov/icfa/

[34] R. Macek (private communication).

[35] E. Perevedentsev (private communication).

[36] L. Wang, H. Fukuma, and K. Ohmi, KEK Report No. 2001-3, 2001.

[37] Y. Suetsugu, Y. Suetsugu, Y. Tanimoto, Y. Hori, M. Kobayashi, and K. Kanazawa, in Proceedings of the International Workshop on Two-Stream Instabilities in Particle Accelerators and Storage Rings, KEK, Tsukuba, Japan, 2001, http://conference.kek.jp/twostream/

[38] Y. Cai, M. Pivi, and M. Furman, SLAC Report No. SLAC-PUB-1-164, 2003.

[39] A. Novokhatski and J. Seeman, in Proceedings of the Particle Accelerator Conference (PAC 03), Portland, Oregon (SLAC Report No. SLAC-PUB-9950, 2003).

[40] R. Macek, CERN Report No. CERN-2002-001, 2002, pp. 259-268. 